\title{
Introducing the Sun and SEPs
}

\begin{abstract}
The structure of the Sun, with its energy generation and heating, creates convection and differential rotation of the outer solar plasma. This convection and rotation of the ionized plasma generates the solar magnetic field. This field and its variation spawn all of the solar activity: solar active regions, flares, jets, and coronal mass ejections (CMEs). Solar activity provides the origin and environment for both the impulsive and gradual solar energetic particle (SEP) events. This chapter introduces the background environment and basic properties of SEP events, time durations, abundances, and solar cycle variations.
\end{abstract}

We tend to think of the Sun as an image of its disk. Recent years have brought increasingly sophisticated images of that disk in the light of single spectral lines and images of active emissions from its surface and its corona with higher and higher spatial resolution. However, we have no such images of solar energetic particles (SEPs). In a photon-dominated discipline, SEPs are stealthy and obscure; they are invisible in the solar sky. While photons travel line-of-sight, SEPs are guided out to us along open magnetic field lines. We must measure, identify, and count SEPs directly one by one. Only in recent years have we overcome the limitations so our observations now begin to bear richer fruit. This is the story of that development.

Solar energetic particles (SEPs) come as bursts of high-energy particles from the direction of the Sun lasting for hours or sometimes days. The particle energies range from about $10 \mathrm{keV}$ (kilo electron volts) to relativistic energies of several $\mathrm{GeV}$, particle speeds $90 \%$ of the speed of light. In addition to the dominant protons and electrons, all of the other chemical elements from $\mathrm{He}$ through $\mathrm{Au}$ and $\mathrm{Pb}$ have now been measured. The relative abundances of these elements and their isotopes have been a powerful new resource in our quest for understanding the physical processes of acceleration and interplanetary transport of SEPs which alter those abundances in distinctive ways. 
In this chapter we introduce properties of SEPs after reviewing some properties of the solar and interplanetary environment in which they are found.

\subsection{The Structure of the Sun}

With a mass of $1.989 \times 10^{33} \mathrm{~g}$, the Sun dominates its neighborhood. It consists of gaseous, ionized plasma where the inner core (see Fig. 1.1) reaches temperatures of 15 million degrees Kelvin (MK) where some of the protons have enough energy to tunnel the Coulomb barrier of the nuclear charge. As they penetrate $\mathrm{H}, \mathrm{C}$, and $\mathrm{N}$ nuclei, they cause the nuclear reactions that catalyze the conversion of $\mathrm{H}$ into ${ }^{4} \mathrm{He}$. The energy released in this process is radiated and reabsorbed as it diffuses outward across the radiative zone, creating sufficient heat and pressure to balance the gravitational force trying to collapse the star.

Circulation of the hot plasma across the convection zone brings energy to the photosphere, that surface where overlying material is too thin to absorb radiation or

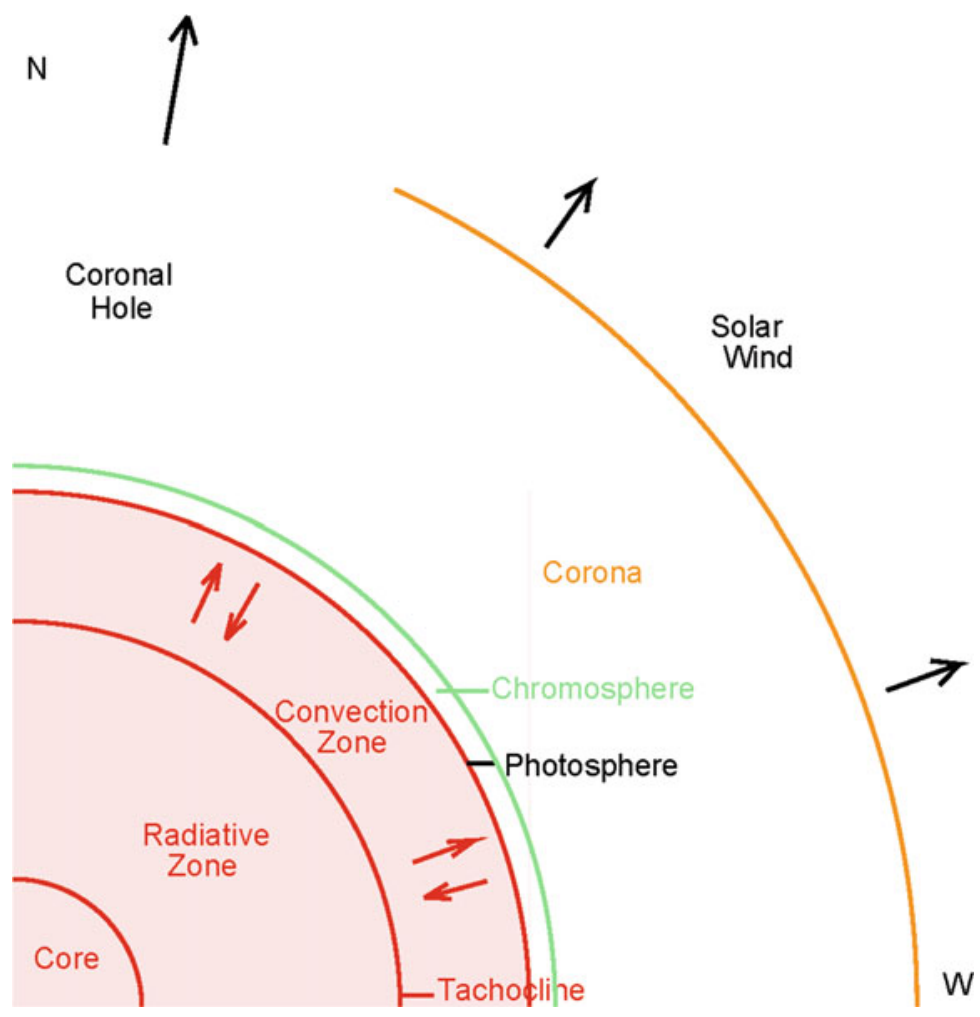

Fig. 1.1 A cross section of the Sun shows its major radial structure from the core to the evaporating solar wind (If we look at the Sun with North at the top and South at the bottom, West is to the right and East to the left. The solar limb is the edge of the visible disk) 
prevent its escape out into space. Here radiation of energy cools the photosphere to $\sim 5800 \mathrm{~K}$ or to $\sim 4500 \mathrm{~K}$ in sunspots which are sites of strong emerging magnetic field. At these temperatures, elements with a first ionization potential (FIP) below about $10 \mathrm{eV}$, just below that of $\mathrm{H}$ at $13.6 \mathrm{eV}$, remain ionized, while those with higher FIP can capture and retain electrons to become neutral atoms.

Above the photosphere lies the narrow chromosphere where the electron temperature $T_{\mathrm{e}}$ remains about $6000 \mathrm{~K}$ over a height of about $2 \mathrm{Mm}$. At its upper boundary, the electron density $n_{\mathrm{e}}$ suddenly falls from $\sim 10^{11} \mathrm{~cm}^{-3}$ to $10^{9} \mathrm{~cm}^{-3}$ and $T_{\mathrm{e}}$ rapidly rises again to over $1 \mathrm{MK}$ in the solar corona (e.g. Aschwanden 2005) which extends outward about another solar radius. The corona is heated either by numerous small sites of magnetic reconnection (nanoflares; Parker 1988) or by absorption of Alfvén waves, plasma waves created in the turbulent layers below, and is largely contained by rising closed magnetic loops. The outer layer of the corona evaporates to become the $400-800 \mathrm{~km} \mathrm{~s}^{-1}$ solar wind which continues to blow past the Earth at $1 \mathrm{AU}$ and far beyond the planets to nearly $100 \mathrm{AU}$. Properties of the solar wind were predicted by Parker (1963) before it was observed.

Inside the tachocline, which lies at the base of the convective zone, the Sun rotates (from East to West) like a rigid body, but throughout the convective zone the Sun rotates differentially, faster at the equator than at the poles. The sidereal period of solar rotation at the equator is 24.47 days but it is $25 \%$ longer at latitude $60^{\circ}$. Azimuthal surfaces of constant rotation-speed run radially through the convection zone forming conical shells about the rotation axis that extend inward only to the tachocline and not to their apex at the center of the Sun.

\subsection{The Solar Magnetic Field}

The Sun has a magnetic field that is generally dipolar in nature, although its origin is still not perfectly understood (see Parker 2009; Sheeley 2005). Magnetic fields, produced in the extreme rotational sheer at the tachocline, are buoyant and produce omega $(\Omega)$ loops that rise through the convection zone and emerge through the photosphere to form sunspots and active regions (Fig. 1.2) as they are sheared and reconnected by the differential rotation. Clusters of magnetic field lines of one polarity tend to emerge from the photosphere at one sunspot and reenter at a nearby spot, leading or following it in the solar rotation. Magnetic fields in sunspots reach 2000-3000 G (0.2-0.3 T). Active regions tend to occur at mid-latitudes on the Sun where the effect of differential rotation on field generation is greatest. When oppositely directed fields reconnect in the largely collisionless regime of the corona, as much as half of the released magnetic energy can be converted to energy of SEPs, with especially copious electrons (Krucker et al. 2010). On closed magnetic loops, this can result in sudden heating and X-ray production in the denser loop footpoints, mainly by electron Bremstrahlung (electron-ion scattering), which is seen as a solar flare (Fletcher et al. 2011). Heating trapped flare plasma to 10-40 MK causes the bright flash of softer radiation. Similar reconnection on open field lines, causing jets (Raouafi et al. 2016), can release electrons and ions into space, i.e. accelerate an 


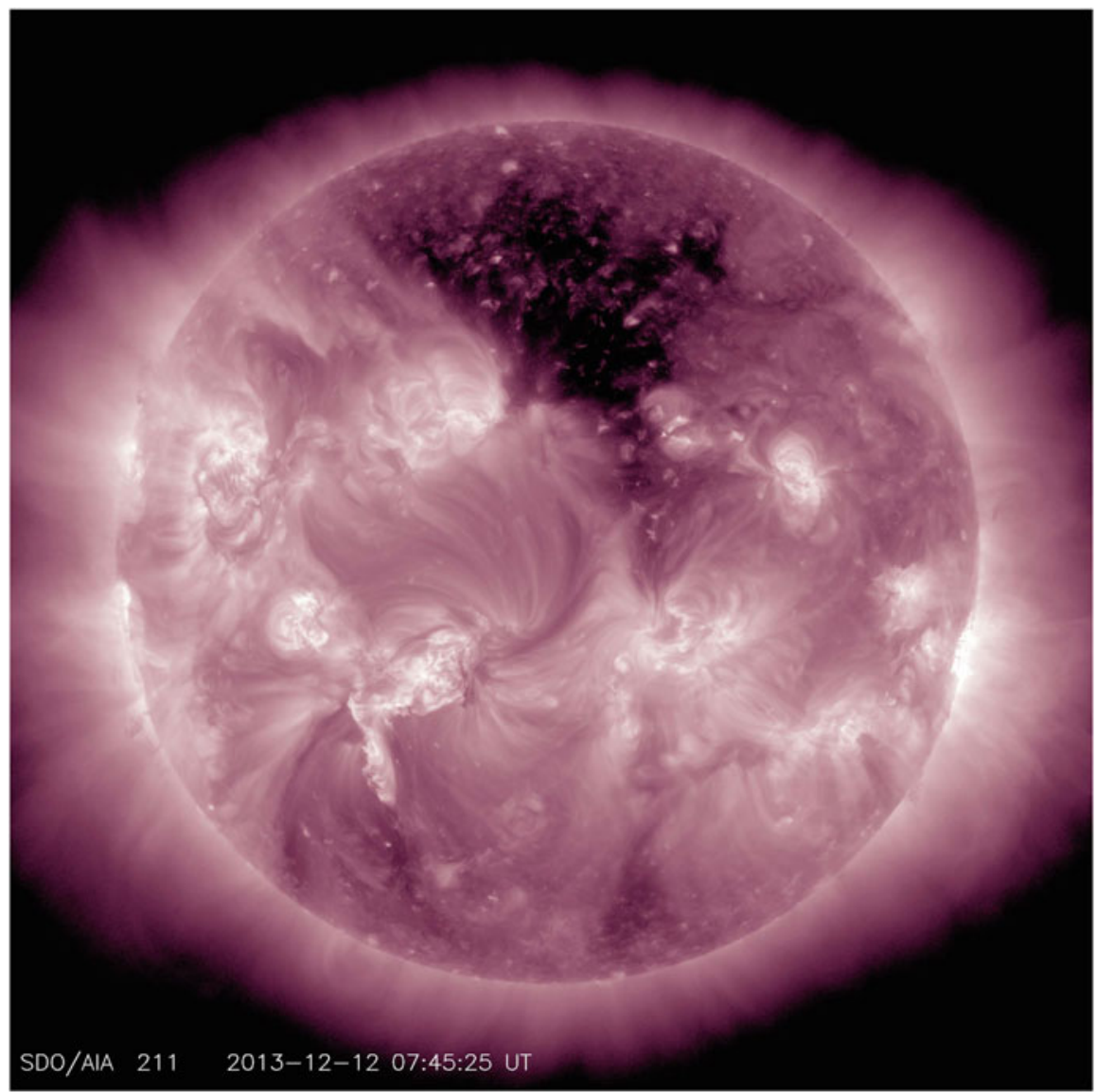

Fig. 1.2 An image of the Sun in $211 \AA$ UV light, taken by the Atmospheric Imaging Assembly on the Solar Dynamics Observatory, shows brightening of magnetically-complex active regions and a large, dark coronal hole

impulsive SEP event, with minimal trapping or heating, as we shall see. As electrons stream out along open field lines they produce fast-drift type-III radio bursts at the local plasma frequency.

As we proceed to smaller and smaller flares, they become more and more numerous as a power law. Parker (1988) suggested that the magnetic reconnection in nanoflares actually provides the energy that heats the solar corona.

Figure 1.2 shows an image of the Sun in ultraviolet (UV) light taken by the Atmospheric Imaging Assembly (AIA) on the NASA spacecraft Solar Dynamics Observatory (SDO; https://sdo.gsfc.nasa.gov/). Complex, bright areas in Fig. 1.2 are active regions while the large dark region on the solar image is a coronal hole. Coronal holes, often seen near the poles, are regions of open magnetic field lines extending into the outer heliosphere, stretched out by the plasma of the solar wind. 
The bright regions show locally closed field lines, i.e. loops, where any accelerated particles are contained and interact so that heating is greatly increased.

Of course, Maxwell's Equations tell us that all magnetic-field lines are closed. However, some field lines are drawn far out into the outer heliosphere by CMEs and the solar wind. For purposes of SEP flow, we describe those field lines as open if they can conduct charged particles out to an observer at or beyond Earth.

The direction of the solar dipolar magnetic field reverses in a cycle of one reversal in about 11 year and solar activity increases as the field reverses. Solar minima occur when the field axis aligns with the solar rotation axis, in one polarity or the other, and the number and size of active regions decreases dramatically. Solar maxima occur during intermediate times and the Sun appears as in Fig. 1.2 late in 2013. During solar minimum the northern hemisphere contains nearly radial field lines of one polarity while the southern hemisphere contains the other; the hemispheres are separated by a plane (or wavy) current sheet, separating the opposite field polarities, extending out into interplanetary space from near the equator. High-speed solar wind $\left(\sim 700-800 \mathrm{~km} \mathrm{~s}^{-1}\right)$ emerges from coronal holes.

The plasma beta, $\beta_{\mathrm{P}}=\rho k T /\left(B^{2} / 8 \pi\right)$, where $\rho$ is the density and $T$ the temperature, is the ratio of thermal to magnetic energy density. When $\beta_{\mathrm{P}}<1$, the field controls the plasma, $B$ is smooth and uniform, and particles are confined to magnetic flux tubes; when $\beta_{\mathrm{P}}>1$, the field becomes variable and distorted by plasma flow and turbulence. The internal structure of CMEs is dominated by magnetic field energy, with $\beta_{\mathrm{P}}<1$.

Most of the solar corona is controlled by magnetic fields with $\beta_{\mathrm{P}}<1$. Plasma can only flow along magnetic loops or flux tubes and cannot escape otherwise. Small neighboring flux tubes can have significantly different values of $T_{\mathrm{e}}$ and $n_{\mathrm{e}}$. However, $\beta_{\mathrm{P}}$ increases with height in the corona and when $\beta_{\mathrm{P}}>1$, plasma is no longer trapped on magnetic loops; it can expand into space, drawing the magnetic fields outward into the solar wind. This tends to defines the "top" of the corona and typically occurs near $2 \mathrm{R}_{\mathrm{S}}$ where $n_{\mathrm{e}} \sim 10^{6} \mathrm{~cm}^{-3}$.

\subsection{Coronal Mass Ejections (CMEs)}

Magnetic reconnection can lead to the ejection of large filaments containing $10^{14}$ $10^{16} \mathrm{~g}$ mass and helical magnetic field with total kinetic energies of $10^{27}-10^{32} \mathrm{ergs}$, carrying most of the energy in solar eruptions (Webb and Howard 2012). CME speeds can be as slow as the solar wind or can exceed $3000 \mathrm{~km} \mathrm{~s}^{-1}$. Figure 1.3 shows a large CME imaged by the Large Angle and Spectrometric Coronagraph (LASCO) on the Solar and Heliospheric Observatory (SOHO; https://sohowww.nascom.nasa. gov/) with a $304 \AA$ image of the Sun from the Extreme Ultraviolet Imaging Telescope (EIT) near the same time scaled onto the coronagraph occulting disk. CME theory and models have been reviewed by Forbes et al. (2006). CMEs only became visible when coronagraphs could block scattered light from the Sun which is $10^{6}$ times brighter. 


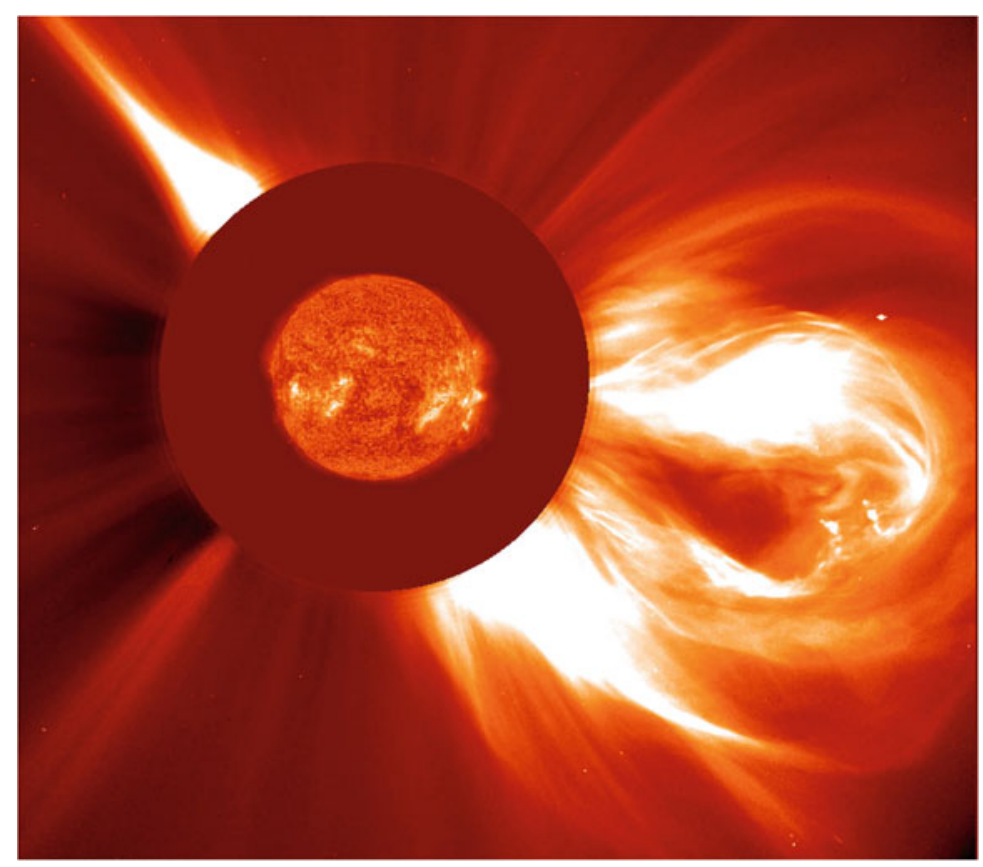

Fig. 1.3 A composite image from the EIT and LASCO telescopes on the NASA/ESA SOHO spacecraft shows a large CME being ejected toward the southwest

Filaments are irregular linear structures of cool, dense, chromospheric plasma magnetically suspended in the corona lying parallel to the solar surface, supported at oppositely-directed magnetic fields beneath an arcade of coronal loops (Martin 1998). They appear dark in $\mathrm{H} \alpha$ solar images and can hang above the photosphere for days. Filaments that project beyond the solar limb are called prominences. Filaments are often ejected as the core of CMEs. In some cases filaments that are present for many days, are suddenly ejected as a CME with no associated flare. These disappearing-filament events can drive shock waves and produce SEPs but they lack an associated flare (see Sect. 2.7). There is also a significant number of "stealth CMEs"; these also have no apparent effect on the lower corona but are also too slow to drive shock waves.

When the speed of a CME exceeds the speed of waves in the plasma of the corona or solar wind, it can drive a collisionless shock wave. We will see that fast, wide CMEs drive shock waves that are the primary source of acceleration of the largest SEP events (Kahler et al. 1984).

A bright streamer is seen in the upper left (northeast) corner of Fig. 1.3, opposite the CME. The streamer belt circles the Sun defining the neutral sheet between fields of opposite polarity. Streamers are the magnetic structures stretched behind CMEs after they move out into the heliosphere. As such, they represent newly opening field lines and may contribute to the slow $\left(\sim 400 \mathrm{~km} \mathrm{~s}^{-1}\right)$ solar wind, although the source 
of the slow solar wind is not fully resolved (e.g. Antiochos et al. 2011). Thus, out-flowing CMEs contribute to the average magnetic field in the heliosphere, which is larger following strong, active solar cycles than weak ones.

\subsection{Interplanetary Space}

The solar wind expands nearly radially outward from the Sun carrying plasma and magnetic field. The solar-wind speed remains approximately constant with distance from the Sun. As the Sun rotates, the field line connected to a given point on its surface is drawn into a spiral pattern, the Parker spiral. In the inner heliosphere, the plasma density and magnetic-field strength decrease approximately as $r^{-2}$ with distance $r$, from the Sun, and as $B \sim r^{-1.5}$ by 1 AU (Burlaga 1995, 2001).

Near Earth the typical magnetic field $B$ is $\sim 10 \mathrm{nT}$, the typical plasma density is $\sim 10$ particles $\mathrm{cm}^{-3}$, and the electron plasma frequency, which varies with the electron density, $n_{\mathrm{e}}$, as $n_{\mathrm{e}}{ }^{1 / 2}$, is $\sim 30 \mathrm{kHz}$. The solar radius, $R_{\mathrm{S}}=6.96 \times 10^{8} \mathrm{~m}=696 \mathrm{Mm}$, and the Earth-Sun distance, $1 \mathrm{AU}$, is $1.50 \times 10^{11} \mathrm{~m}=216 R_{\mathrm{S}}$, often a useful number. In this spirit, plasma in the $400 \mathrm{~km} \mathrm{~s}^{-1}$ solar wind takes 4.3 days to travel $1 \mathrm{AU}$, a shock wave with an average speed of $1700 \mathrm{~km} \mathrm{~s}^{-1}$ takes one day, a $10 \mathrm{MeV}$ proton or a $5 \mathrm{keV}$ electron takes an hour, and a photon of light takes $8.3 \mathrm{~min}$. Thus, it is not surprising that particles accelerated by a shock wave near the Sun arrive near Earth long before the arrival of the shock itself.

Alfvén waves propagate through plasma with correlated variations in $B$ and the plasma density $\rho$ with a speed $V_{\mathrm{A}}=B /(4 \pi \rho)^{1 / 2}$. In models of $V_{\mathrm{A}}$ in the solar atmosphere above an active region (e.g. Mann et al. 2003), $V_{\mathrm{A}}$ falls rapidly with height to a value of $\sim 200-500 \mathrm{~km} \mathrm{~s}^{-1}$ at $r \approx 1.5 R_{S}$, it then rises to a broad maximum of $\sim 750 \mathrm{~km} \mathrm{~s}^{-1}$ near $4 R_{\mathrm{S}}$ and finally decays approximately as $r^{-1}$ out toward Earth (Mann et al. 2003) where it is nominally $30 \mathrm{~km} \mathrm{~s}^{-1}$. However, these values depend upon assumptions about the magnetic structure of an active region. The behavior of $V_{\mathrm{A}}$ is important since the disturbance caused by a CME must exceed the speed of Alfvén waves to form a shock wave which can accelerate SEPs.

Large CMEs can be recognized in the solar wind when they pass Earth (often called ICMEs) and lists of them, with their associated coronagraphic origin, have been published (Richardson and Cane 2010). A class of particularly regular events called magnetic clouds is identified by a flux-rope magnetic field that spirals slowly through a large angle (Burlaga et al. 1981). Shock waves driven out by CMEs can also be observed near Earth and their properties can be determined (e.g. Berdichevsky et al. 2000). Lists of properties of interplanetary shock waves spanning many years (since 1995) are available for shocks observed at the Wind and ACE (Advanced Composition Explorer) spacecraft (https://www.cfa.harvard.edu/ shocks/). We will see examples of shock waves later in this book. 


\subsection{Solar Energetic Particles}

Energetic charged particles must be accelerated in nearly collisionless plasma at high coronal altitudes so they do not lose all their new energy in Coulomb collisions. Particles accelerated at the tops of magnetic loops will soon scatter through the loss cone into the footpoints of those loops where they deposit their energy in much higher ambient densities, producing a solar flare. The rate of heating and production of photons generally depends upon the product of energetic-particle intensity times the ambient particle density. Thus SEP acceleration sites tend to be barely visible, high in the corona, while most of the photons are produced low in the corona. Looking only at photons, we do not see where these SEPs are "born"; we see where they "die". Especially troublesome is shock acceleration at $\sim 2$ solar radii and beyond, where SEPs in the acceleration site are completely invisible. This inconvenient fact has led to great confusion about the origin of SEPs.

The effort to understand the physical origin of SEP events finally led to the identification of two classes of SEP events, impulsive and gradual (or long-duration) with the sources suggested by Fig. 1.4 (e.g. Reames 1999, 2013). The history of this journey will be discussed in Chap. 2 with further physical evidence in Chap. 3. Fortunately, the properties of the SEPs themselves carry the imprint of both their origin and their transport. Important differences lie in abundances of elements and isotopes, electron/ion ratios, energy spectra, onset timing, duration, angular distributions, and associations with visible phenomena, as we shall see.

The data base for many measurements from many spacecraft, including SEP intensities, from spacecraft where they were measured, is the NASA Coordinated Data and Analysis Web site: https://cdaweb.gsfc.nasa.gov/sp_phys/. This web site has data from past and current space-physics missions.

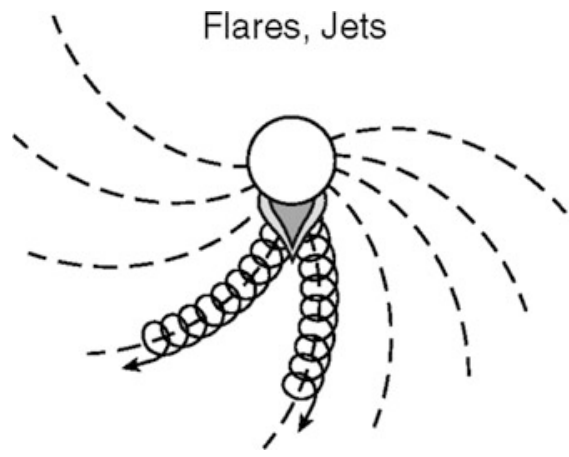

\section{CME Shocks}

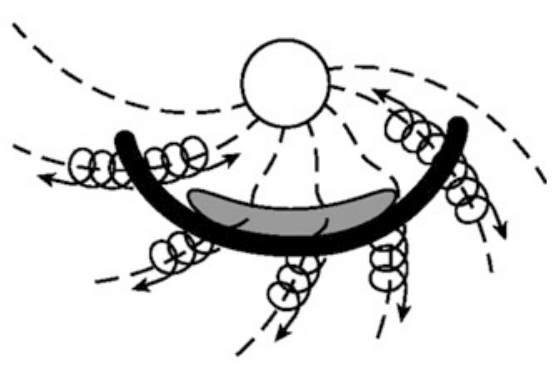

Fig. 1.4 Impulsive (left) and gradual (right) classes of SEP events are distinguished by the dominant sources of particle acceleration in each case (Reames 1999, (C) Springer). Impulsive SEP events are accelerated in magnetic-reconnection events on open field lines (i.e. jets) in the corona. Gradual SEP events are accelerated at shock waves (solid black) driven out from the Sun by CMEs (gray). Particle trajectories are shown as spirals along $\boldsymbol{B}$ (dashed) 


\subsubsection{Time Duration}

While the terms impulsive and gradual did not originally refer to the SEP duration, this is often a reasonable characterization, as shown by the events in Fig. 1.5.

\subsubsection{Abundances}

The abundances of elements and isotopes have been powerful indicators of the origin, acceleration, and transport of SEPs. It was found (Webber 1975; Meyer 1985 ) that the average element abundances, in events we now call large, gradual SEP events, were a measure of the corresponding solar coronal abundances. These differ from abundances in the photosphere by a factor which depends on the first ionization potential (FIP) of the element as shown in Fig. 1.6 and listed in Table 1.1 (Reames 1995, 2014). In the photosphere, low-FIP $(<10 \mathrm{eV})$ elements are ionized while highFIP elements are neutral atoms. Relative to the upward flow of neutral atoms, ions are also influenced by Alfvén waves during their transport across the solar chromosphere and into the corona (e.g. Laming 2009, 2015). Other measures of coronal abundances, such as in the solar wind (e.g. Geiss 1982; Bochsler 2009), show a FIP effect that is similar but not identical to SEPs (see Sect. 8.4; Schmelz et al. 2012; Reames 2018), reflecting a different origin. The SEP abundances in Table 1.1 can serve as reference abundances for discussion of "enhancements" throughout this book.

Table 1.1 lists the photospheric (Asplund et al. 2009) and the reference SEP (Reames 1995, 2014, 2020) abundances that we can use. A likely correction to the
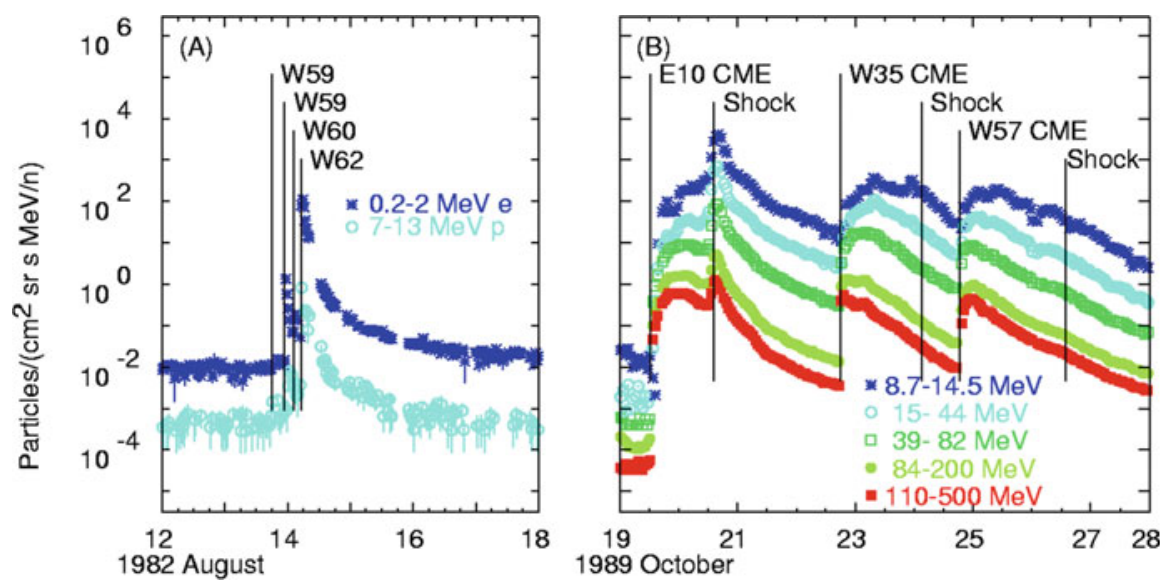

Fig. 1.5 Particle intensities are shown for a series of (a) impulsive and (b) gradual or long-duration SEP events at similar time and intensity scales. Flags labeled with the source longitude indicate the onset times of the events; also shown are the times of shock passage near Earth. Proton (or electron) energies are listed. It is difficult to obtain comparable proton energies because impulsive events are much less energetic (Reames 1999 (C) Springer) 


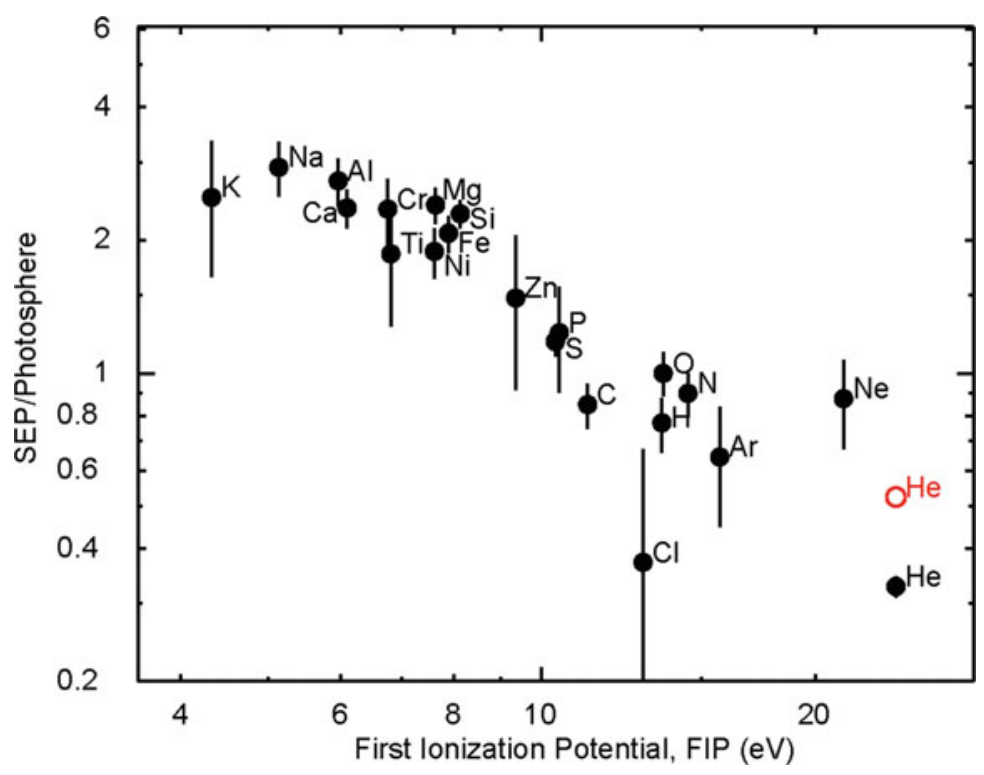

Fig. 1.6 The average element abundance in gradual SEP events (Reames 1995, 2014, 2017), or reference abundance, relative to the corresponding abundance in the solar photosphere (Asplund et al. 2009) is plotted as a function of the FIP of the element (see text). Here He means ${ }^{4} \mathrm{He}$

reference abundance of $\mathrm{He}(\mathrm{He} / \mathrm{O}=91$ rather than 57; Reames 2017), that will be discussed in Sect. 5.9, is shown as a red open circle in Fig. 1.6. Alternative photospheric abundances by Caffau et al. (2011), shown in Table 8.1, make some difference in the FIP plot as demonstrated by Reames (2015); the differences depend upon the choice of spectral lines used to obtain the photospheric abundance measurements (Chap. 8). Note that within this book, unspecified He means ${ }^{4} \mathrm{He}$.

The current theory of the "FIP effect" (Laming 2009, 2015; Laming et al. 2019) uses the ponderomotive force of Alfvén waves below the chromospheric-coronal boundary to preferentially boost low-FIP ions (e.g. $\mathrm{Mg}, \mathrm{Si}, \mathrm{Fe}$ ) into the corona ahead of the slower evaporation of high-FIP neutral atoms (e.g. O, Ne, He). All elements become ionized in the 1-MK corona. The Alfvén waves can resonate with the loop length on closed magnetic loops and calculations show that the ion fractionation only occurs near the top of the chromosphere. Open fields lack this resonance so the fractionation is more extensive. Particles destined to become SEPs apparently arise mainly on closed loops in active regions and the elements $\mathrm{P}, \mathrm{S}$, and $\mathrm{C}$ are suppressed like high-FIP neutral atoms, while particles of the solar wind arise on open field lines where P, S, and C behave like low-FIP ions (see Sect. 5.9, Chap. 8, and Reames 2018, 2020). This means that SEPs are not merely accelerated solar wind, as originally noticed by Mewaldt et al. (2002) and Desai et al. (2003); SEPs and the solar wind are fundamentally different samples of coronal material. The FIP patterns of SEPs and the solar wind are compared with theory in Chap. 8 and the differing locations of their origin are discussed. 
Table 1.1 Photospheric and SEP-reference abundances used in Fig. 1.6

\begin{tabular}{l|l|l|l|l}
\hline & $\mathrm{Z}$ & FIP $[\mathrm{eV}]$ & Photosphere & SEP Reference \\
\hline $\mathrm{H}$ & 1 & 13.6 & $(2.04 \pm 0.05) \times 10^{6}$ & $(\sim 1.57 \pm 0.22) \times 10^{6}$ \\
\hline $\mathrm{He}$ & 2 & 24.6 & $(1.74 \pm 0.04) \times 10^{5}$ & $57,000 \pm 3000,91,000 \pm 13,000$ \\
\hline $\mathrm{C}$ & 6 & 11.3 & $550 \pm 63$ & $420 \pm 10$ \\
\hline $\mathrm{N}$ & 7 & 14.5 & $138 \pm 16$ & $128 \pm 8$ \\
\hline $\mathrm{O}$ & 8 & 13.6 & $1000 \pm 115$ & $1000 \pm 10$ \\
\hline $\mathrm{Ne}$ & 10 & 21.6 & $174 \pm 40$ & $157 \pm 10$ \\
\hline $\mathrm{Na}$ & 11 & 5.1 & $3.55 \pm 0.33$ & $10.4 \pm 1.1$ \\
\hline $\mathrm{Mg}$ & 12 & 7.6 & $81 \pm 8$ & $178 \pm 4$ \\
\hline $\mathrm{Al}$ & 13 & 6.0 & $5.75 \pm 0.40$ & $15.7 \pm 1.6$ \\
\hline $\mathrm{Si}$ & 14 & 8.2 & $66.1 \pm 4.6$ & $151 \pm 4$ \\
\hline $\mathrm{P}$ & 15 & 10.5 & $0.525 \pm 0.036$ & $0.65 \pm 0.17$ \\
\hline $\mathrm{S}$ & 16 & 10.4 & $26.9 \pm 1.9$ & $25 \pm 2$ \\
\hline $\mathrm{Cl}$ & 17 & 13.0 & $0.65 \pm 0.45$ & $0.24 \pm 0.1$ \\
\hline $\mathrm{Ar}$ & 18 & 15.8 & $5.1 \pm 1.5$ & $4.3 \pm 0.4$ \\
\hline $\mathrm{K}$ & 19 & 4.3 & $0.22 \pm 0.14$ & $0.55 \pm 0.15$ \\
\hline $\mathrm{Ca}$ & 20 & 6.1 & $4.47 \pm 0.41$ & $11 \pm 1$ \\
\hline $\mathrm{Ti}$ & 22 & 6.8 & $0.182 \pm 0.021$ & $0.34 \pm 0.1$ \\
\hline $\mathrm{Cr}$ & 24 & 6.8 & $0.89 \pm 0.08$ & $2.1 \pm 0.3$ \\
\hline $\mathrm{Fe}$ & 26 & 7.9 & $64.6 \pm 6.0$ & $131 \pm 6$ \\
\hline $\mathrm{Ni}$ & 28 & 7.6 & $3.39 \pm 0.31$ & $6.4 \pm 0.6$ \\
\hline $\mathrm{Zn}$ & 30 & 9.4 & $0.074 \pm 0.009$ & $0.11 \pm 0.04$ \\
\hline & & & &
\end{tabular}

Abundances also distinguish impulsive SEP events in a very different way (Mason 2007). The earliest of these was the greatly enhanced ${ }^{3} \mathrm{He} /{ }^{4} \mathrm{He}$ ratio, which is $\sim 5 \times 10^{-4}$ in the solar wind, but can be $>1$ in impulsive SEP events, as seen in the examples in Fig. 1.7. Enhancements of Fe/O by a factor of $\sim 10$ were subsequently observed and we now see these as part of an enhancement that is a power law in $A / Q$ that becomes a 1000 -fold enhancement for heavy elements up to $\mathrm{Au}$ and $\mathrm{Pb}$, relative to $\mathrm{He}$ or $\mathrm{O}$. These orderly systematic enhancements in impulsive SEP events will be discussed in Chap. 4. The observation of energy-dependent stripping of electrons from $\mathrm{Fe}$ ions after acceleration now suggests they were accelerated at or below 1.5 solar radii (DiFabio et al. 2008) in solar jets (see Sect 2.6).

The two events in Fig. 1.7 have event-averaged $\mathrm{Fe} / \mathrm{O}=1.24 \pm 0.28$ and $1.34 \pm 0.20$, respectively, compared with the reference value of $0.131 \pm 0.006$ in Table 1.1. Enhancements of even heavier elements (e.g. $Z>50$ ) are much greater, on average, but are difficult to measure in single small events. These will be seen in Sect. 4.5.

Impulsive SEP events are associated with solar jets (Bučík 2020), i.e. magnetic reconnection on open field lines that allow the SEPs and narrow CMEs to escape (Kahler et al. 2001), especially those more-energetic jets from solar active regions (Bučík et al. 2018a, b). Impulsive SEP events also have intense electron beams 


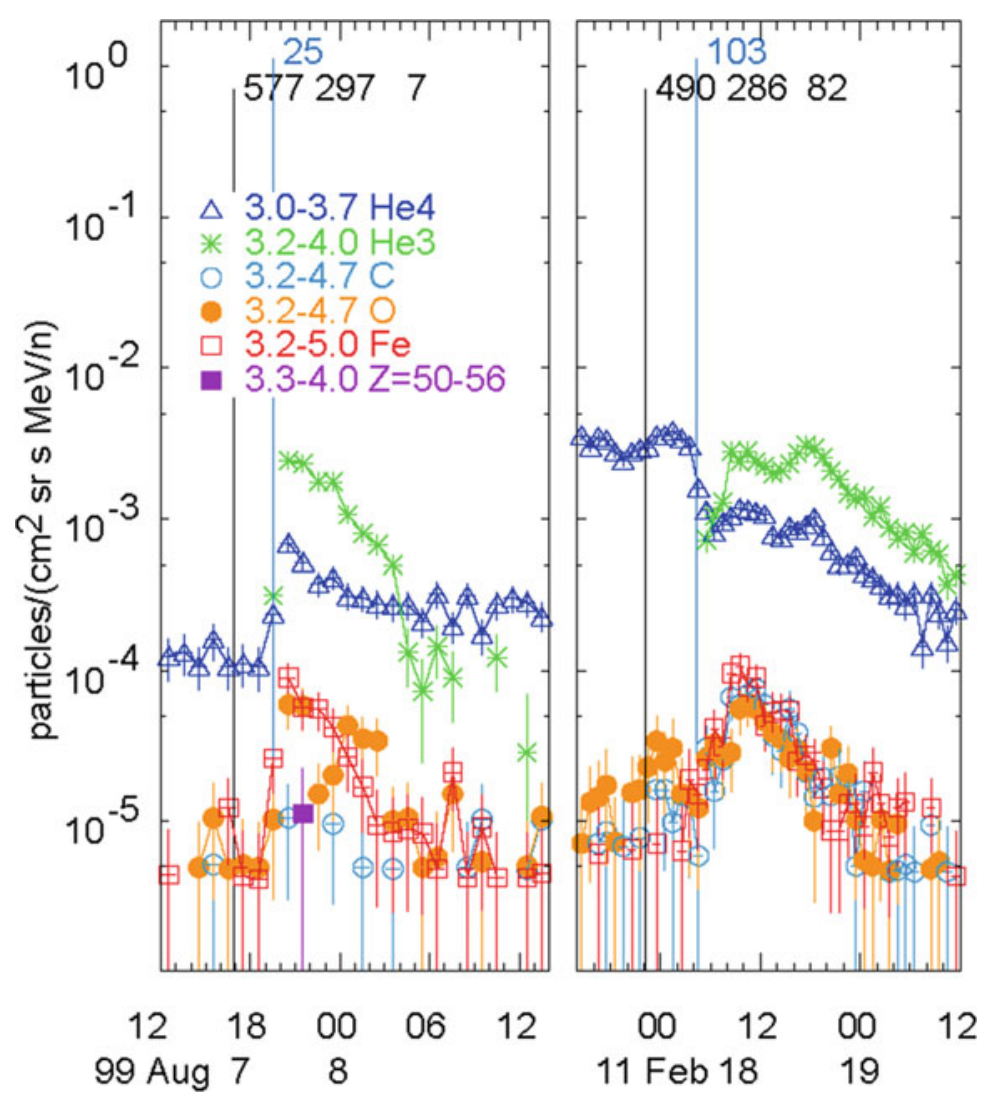

Fig. 1.7 Intensities vs. time are shown in impulsive SEP event numbers 25 and 103 (shown in blue flags at event onsets) from the list in Reames et al. (2014 (C) Springer). ${ }^{3} \mathrm{He}$ exceeds ${ }^{4} \mathrm{He}$ in these events and Fe exceeds $\mathrm{C}$ and $\mathrm{O}$. Flags in black preceding the SEP onsets are at the associated CME onset times and list the speed $\left(\mathrm{km} \mathrm{s}^{-1}\right)$, position angle (deg), and width (deg) of the CME.

(Reames et al. 1985) that emit type III radio bursts (Sect. 2.2; Reames and Stone 1986).

\subsubsection{The Solar Cycle}

SEP events do not precisely follow the solar activity level of sunspots, but they do have a definite solar cycle. The upper panel of Fig. 1.8 shows intensities of 120-230 MeV protons measured by the Goddard Space Flight Center telescope on the IMP-8 (Interplanetary Monitoring Platform) spacecraft. This telescope is sensitive to energetic protons of both solar and galactic origin and can thus observe the counter-cyclical behavior. When the Sun is active with SEP events, the greater ejection of CMEs increases the modulation that blocks and decreases the 


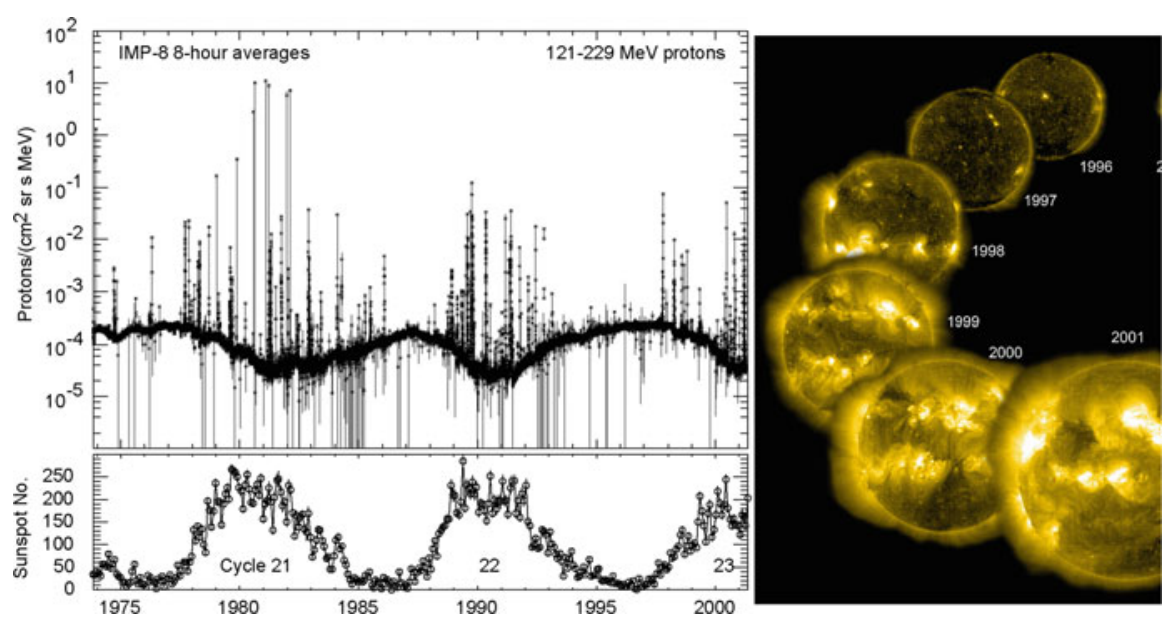

Fig. 1.8 Intensities of $120-230 \mathrm{MeV}$ protons in 8 -h averages from the Goddard IMP-8 telescope are shown over 27 years in the upper panel. Spikes from individual SEP events reach a factor of $10^{5}$ above a counter-cyclical baseline of galactic cosmic rays which the instrument also measures well. However, intensities in some SEP events during the later cycles are foreshortened because of increasingly frequent data gaps. The monthly international sunspot number is shown in the lower panel for comparison, and to the right are EIT images at $284 \AA$ that show solar activity during each year from 1996 to 2001 during the rise of Solar Cycle 23

encroachment of galactic cosmic rays into the heliosphere. The monthly sunspot number is shown in the lower panel for comparison (see Hathaway 2010).

\subsubsection{Relativistic Kinematics}

What we often call the particle "energy," $E$, commonly quoted as $\mathrm{MeV} \mathrm{amu}^{-1}$, is actually a measure of velocity $E=\varepsilon / A=M_{u}(\gamma-1) \approx 1 / 2 M_{\mathrm{u}} \beta^{2}$, where $\varepsilon$ is the total kinetic energy, $A$ is the atomic mass, $M_{u}=m_{u} c^{2}=931.494 \mathrm{MeV}, \gamma=\left(1-\beta^{2}\right)^{-1 / 2}$, and $\beta=v / c$ is the particle velocity relative to the speed of light, $c$. Abundances of elements and isotopes are always compared at the same value of $E$. The total energy of a particle is $W=A M_{u} \gamma$ and the momentum is given by $p c=A M_{u} \beta \gamma$. The magnetic rigidity or momentum per unit charge is $P=p c / Q e=M_{u} \beta \gamma A / Q$ in units of $\mathrm{MV}$. Note that the standard atomic mass unit (amu), $1 / 12$ the mass of ${ }^{12} \mathrm{C}$, is close enough to nucleon masses that $\mathrm{MeV}$ nucleon $^{-1}$ is indistinguishable from $\mathrm{MeV}$ $\mathrm{amu}^{-1}$ for SEP studies.

We can write the Lorentz force on a single particle in the form

$$
m_{u} \frac{d}{d t}(\gamma \mathbf{v})=\frac{Q}{A} e(\mathbf{E}+\mathbf{v} \times \mathbf{B})
$$


In a collisionless world where the electric and magnetic fields are independent of the nature of the particle, the only specific particle species dependence is $Q / A$. This will be the case for most of the wave-particle interactions we will encounter during particle acceleration and transport. The exception comes when the particle interacts with matter where the electric field $\mathbf{E}$ is that of the particle itself and depends upon $Q$ as it scatters electrons of the stopping material. This is the case in particle detectors (Chap. 7) where the species-dependence for energy loss becomes $Q^{2} / A$. Strong enhancements observed in elements with $76 \leq \mathrm{Z} \leq 82$ in impulsive SEPs would have been suppressed by this dependence on $Q^{2} / A$ if the ions had traversed significant amounts of matter during acceleration or transport. Thus, acceleration and transport are primarily collisionless and depend upon $Q / A$, although, in some cases, ions may traverse enough material after acceleration to alter their ionization states (Sect. 2.6).

\subsection{What Do We "See" at the Sun?}

SEPs follow magnetic fields out from the Sun so the image of their source location is distorted and difficult to follow. Are there photons emitted that can help us locate SEP origins?

Many of the energetic photons we see from the Sun are produced by heating. Solar spectral lines that can image the Sun are a specific wavelength produced by ions of a specific element in a specific ionization state, i.e. temperature, of the ambient solar plasma. They show regions of heating and can indicate element abundances in the ambient plasma, but tell us little of the nature of any SEPs that may have caused the heating as they plunged into the denser plasma.

CMEs are imaged in white light reflected by ejected material. It is sunlight from the photosphere that has been Thomson-scattered by free electrons in the corona. The light intensity is a measure of electron density, and coronagraph images of fast CMEs can distinguish the location and evolution of shock waves and the driver gas (see Sect. 3.2).

Hard X-rays ( $>20 \mathrm{keV}$ ) are produced by Bremstrahlung (electron-ion scattering) of energetic electrons. Images show locations where energized $(>20 \mathrm{keV})$ electrons encounter regions of denser plasma (Fletcher et al. 2011; Miller et al. 1997). Soft $\mathrm{X}$-rays are produced in relatively dense regions by electron and ion heating as they stop and lose their energy to the plasma by Coulomb scattering. Thus flares are produced by SEP-heated plasma, but those SEPs don't get out. Magnetic trapping is the reason flares are so hot and bright.

Radio emission is produced by $10-100 \mathrm{keV}$ electrons. Type III bursts are produced by electrons streaming out from the Sun along magnetic field lines while type II bursts are produced at interplanetary shock waves (see Sect. 2.2). 
The only photons identified with accelerated ions are in the broad $\gamma$-ray lines produced by Doppler-shifted emission of interacting energetic ions of the "beam," while the narrow $\gamma$-ray lines are emitted from the ambient plasma in flares (see Sect. 4.9). The last measurements of solar $\gamma$-ray lines were made in the $1980 \mathrm{~s}$. These are the only measurable photons produced by the energetic ions.

It is also possible to trace field lines of direct measurements of SEP events back to the Sun to locate their source (Nitta et al. 2006; Wang et al. 2006; Ko et al. 2013).

Thus, the photons we measure are usually produced by electrons and rarely help us study accelerated ions, so, much of the SEP physics must be determined directly from measurements of the ions themselves. Associations are generally made from models or from similarities in the timing, although the photons usually relate to SEPs accelerated on closed field lines while the SEP ions and electrons we see in space come from related events on open field lines.

Acknowledgements The author thanks the SOHO and SDO projects for figures used in this chapter.

\section{References}

Antiochos, S.K., Mikić, S., Titov, V.S., Lionello, R., Linker, J.A.: A Model for the sources of the slow solar wind. Astrophys. J. 731, 112 (2011). https://doi.org/10.1088/0004-637X/731/2/112

Aschwanden, M.: Physics of the solar corona, Springer, Berlin (2005) ISBN 3-540-30765-6

Asplund, M., Grevesse, N., Sauval, A.J., Scott, P.: The chemical composition of the sun. Annu. Rev. Astron. Astrophys. 47, 481 (2009). https://doi.org/10.1146/annurev.astro.46.060407. 145222

Berdichevsky, D.B., Szabo, A., Lepping, R.P., Vinas, A.F., Mariana, F.: Interplanetary fast shocks and associated drivers observed through the 23rd solar minimum by Wind over its first 2.5 years. J. Geophys. Res. 105, 27289 (2000) doi: https://doi.org/10.1029/1999JA000367; Errata in J. Geophys. Res. 106, 25133 (2001). doi: https://doi.org/10.1029/2001JA000074

Bochsler, P.: Composition of matter in the heliosphere. Proc. Int. Astron. Union Sympos. 257, 17 (2009). https://doi.org/10.1017/S1743921309029044

Bučík, R.: ${ }^{3}$ He-rich solar energetic particles: solar sources. Space Sci. Rev. 216, 24 (2020). https:// doi.org/10.1007/s11214-020-00650-5

Bučík, R., Innes, D.E., Mason, G.M., Wiedenbeck, M.E., Gómez-Herrero, R., Nitta, N.V.: ${ }^{3}$ He-rich solar energetic particles in helical jets on the Sun. Astrophys. J. 852, 76 (2018a). https://doi.org/ 10.3847/1538-4357/aa9d8f

Bučík, R., Wiedenbeck, M.E., Mason, G.M., Gómez-Herrero, R., Nitta, N.V., Wang, L.: ${ }^{3}$ He-rich solar energetic particles from sunspot jets. Astrophys. J. Lett. 869, L21 (2018b). https://doi.org/ 10.3847/2041-8213/aaf37f

Burlaga, L.F.: Interplanetary magnetohydrodynamics, Oxford University Press, Oxford (1995) ISBN 13: 978-0-19-508472-6

Burlaga, L.F.: Magnetic fields and plasmas in the inner heliosphere: Helios results. Planet. Space Sci. 49, 1619 (2001). https://doi.org/10.1016/S0032-0633(01)00098-8

Burlaga, L.F., Sittler, E., Mariani, F., Schwenn, R.: Magnetic loop behind an interplanetary shock: Voyager, Helios, and Imp 8 observations. J. Geophys. Res. 86, 6673 (1981). https://doi.org/10. 1029/JA086iA08p06673 
Caffau, E., Ludwig, H.-G., Steffen, M., Freytag, B., Bonofacio, P.: Solar chemical abundances determined with a CO5BOLD 3D model atmosphere. Sol. Phys. 268, 255 (2011). https://doi. org/10.1007/s11207-010-9541-4

Desai, M.I., Mason, G.M., Dwyer, J.R., Mazur, J.E., Gold, R.E., Krimigis, S.M., Smith, C.W., Skoug, R.M.: Evidence for a suprathermal seed population of heavy ions accelerated by interplanetary shocks near 1 AU. Astrophys. J. 588, 1149 (2003). https://doi.org/10.1086/ 374310

DiFabio, R., Guo, Z., Möbius, E., Klecker, B., Kucharek, H., Mason, G.M., Popecki, M.: Energydependent charge states and their connection with ion abundances in impulsive solar energetic particle events. Astrophys. J. 687, 623 (2008). https://doi.org/10.1086/591833

Fletcher, L., Dennis, B.R., Hudson, H.S., Krucker, S., Phillips, K., Veronig, A., Battaglia, M., Bone, L., Caspi, A., Chen, Q., et al.: An observational view of solar flares. Space Sci. Rev. 159, 19 (2011). https://doi.org/10.1007/s11214-010-9701-8

Forbes, T.G., Linker, J.A., Chen, J., Cid, C., Kóta, J., Lee, M.A., Mann, G., Mikic, Z., Potgieter, M. S., Schmidt, J.M., Syscoe, G.L., Vainio, R., Antiochos, S.K., Riley, P.: CME theory and models. Space Sci. Rev. 123, 251 (2006). https://doi.org/10.1007/s11214-006-9019-8

Geiss, J.: Processes affecting abundances in the solar wind. Space Sci. Rev. 33, 201 (1982). https:// doi.org/10.1007/BF00213254

Hathaway, D.H.: The solar cycle. Living Rev. Sol. Phys. 7, 1 (2010). https://doi.org/10.12942/lrsp2010-1

Kahler, S.W., Sheeley Jr., N.R., Howard, R.A., Koomen, M.J., Michels, D.J., McGuire, R.E., von Rosenvinge, T.T., Reames, D.V.: Associations between coronal mass ejections and solar energetic proton events. J. Geophys. Res. 89, 9683 (1984). https://doi.org/10.1029/ JA089iA11p09683

Kahler, S.W., Reames, D.V., Sheeley Jr., N.R.: Coronal mass ejections associated with impulsive solar energetic particle events. Astrophys. J. 562, 558 (2001). https://doi.org/10.1086/323847

Ko, Y.-K., Tylka, A.J., Ng, C.K., Wang, Y.-M., Dietrich, W.F.: Source regions of the interplanetary magnetic field and variability in heavy-ion elemental composition in gradual solar energetic particle events. Astrophys. J. 776, 92 (2013). https://doi.org/10.1088/0004-637X/776/2/92

Krucker, S., Hudson, H.S., Glesener, L., White, S.M., Masuda, S., Wuelser, J.P., Lin, R.P.: Measurements of the coronal acceleration region of a solar flare. Astrophys. J. 714, 1108 (2010). https://doi.org/10.1088/0004-637X/714/2/1108

Laming, J.M.: Non-WKB models of the first ionization potential effect: implications for solar coronal heating and the coronal helium and neon abundances. Astrophys. J. 695, 954 (2009). https://doi.org/10.1088/0004-637X/695/2/954

Laming, J.M.: The FIP and inverse FIP effects in solar and stellar coronae. Living Rev. Sol. Phys. 12, 2 (2015). https://doi.org/10.1007/lrsp-2015-2

Laming, J.M., Vourlidas, A., Korendyke, C., et al.: Element abundances: a new diagnostic for the solar wind. Astrophys. J. 879124 (2019) doi: https://doi.org/10.3847/1538-4357/ab23f1 arXiv: 19005.09319

Mann, G., Klassen, A., Aurass, H., Classen, H.-T.: Formation and development of shock waves in the solar corona and the near-Sun interplanetary space. Astron. Astrophys. 400, 329 (2003). https://doi.org/10.1051/0004-6361:20021593

Martin, S.F.: Conditions for the formation and maintenance of filaments (invited review). Solar Phys. 182, 126 (1998). https://doi.org/10.1023/A:1005026814076

Mason, G.M.: ${ }^{3}$ He-rich solar energetic particle events. Space Sci. Rev. 130, 231 (2007). https://doi. org/10.1007/s11214-007-9156-8

Mewaldt, R.A., Cohen, C.M.S., Leske, R.A., Christian, E.R., Cummings, A.C., Stone, E.C., von Rosenvinge, T.T., Wiedenbeck, M.E.: Fractionation of solar energetic particles and solar wind according to first ionization potential. Advan. Space Res. 30, 79 (2002). https://doi.org/10.1016/ S0273-1177(02)00263-6

Meyer, J.P.: The baseline composition of solar energetic particles. Astrophys. J. Suppl. 57, 151 (1985). https://doi.org/10.1086/191000 
Miller, J.A., Cargill, P.J., Emslie, A.G., Holman, G.D., Dennis, B.R., LaRosa, T.N., Winglee, R.M., Benka, S.G., Tsuneta, S.: Critical issues for understanding particle acceleration in impulsive solar flares. J. Geophys. Res. 102, 14631 (1997). https://doi.org/10.1029/97JA00976

Nitta, N.V., Reames, D.V., DeRosa, M.L., Yashiro, S., Gopalswamy, N.: Solar sources of impulsive solar energetic particle events and their magnetic field connection to the earth. Astrophys. J. 650, 438 (2006). https://doi.org/10.1086/507442

Parker, E.N.: Interplanetary dynamical processes. Interscience, New York (1963)

Parker, E.N.: Nanoflares and the solar X-ray corona. Astrophys. J. 330, 474 (1988). https://doi.org/ $10.1086 / 166485$

Parker, E.N.: Solar magnetism: the state of our knowledge and ignorance. Space Sci. Rev. 144, 15 (2009). https://doi.org/10.1007/s11214-008-9445-X

Raouafi, N.E., Patsourakos, S., Pariat, E., Young, P.R., Sterling, A.C., Savcheva, A., Shimojo, M., Moreno-Insertis, F., DeVore, C.R., Archontis, V., et al.: Solar coronal jets: observations, theory, and modeling. Space Sci. Rev. 201, 1 (2016). https://doi.org/10.1007/s11214-016-0260-5. (arXiv:1607.02108)

Reames, D.V.: Coronal abundances determined from energetic particles. Adv. Space Res. 15(7), 41 (1995)

Reames, D.V.: Particle acceleration at the Sun and in the Heliosphere. Space Sci. Rev. 90, 413 (1999). https://doi.org/10.1023/A:1005105831781

Reames, D.V.: The two sources of solar energetic particles. Space Sci. Rev. 175, 53 (2013). https:// doi.org/10.1007/s11214-013-9958-9

Reames, D.V.: Element abundances in solar energetic particles and the solar corona. Sol. Phys. 289, 977 (2014). https://doi.org/10.1007/s11207-013-0350-4

Reames, D.V.: What are the sources of solar energetic particles? Element abundances and source plasma temperatures. Space Sci. Rev. 194, 303 (2015). https://doi.org/10.1007/s11214-0150210-7

Reames, D.V.: The abundance of helium in the source plasma of solar energetic particles. Sol. Phys. 292, 156 (2017). https://doi.org/10.1007/s11207-017-1173-5. (arXiv: 1708.05034)

Reames, D.V.: The "FIP effect" and the origins of solar energetic particles and of the solar wind. Sol. Phys. 294, 47 (2018). https://doi.org/10.1007/s11207-018-1267-8. (arXiv: 1801.05840)

Reames, D.V.: Four distinct pathways to the element abundances in solar energetic particles. Space Sci. Rev. 216, 20 (2020). https://doi.org/10.1007/s11214-020-0643-5. (arXiv: 1912.06691)

Reames, D.V., Stone, R.G.: The identification of solar He-3-rich events and the study of particle acceleration at the sun. Astrophys. J. 308, 902 (1986). https://doi.org/10.1086/164560

Reames, D.V., von Rosenvinge, T.T., Lin, R.P.: Solar He-3-rich events and nonrelativistic electron events - a new association. Astrophys. J. 292, 716 (1985). https://doi.org/10.1086/163203

Reames, D.V., Cliver, E.W., Kahler, S.W.: Abundance enhancements in impulsive solar energeticparticle events with associated coronal mass ejections. Sol. Phys. 289, 3817 (2014). https://doi. org/10.1007/s11207-014-0547-1

Richardson, I.G., Cane, H.V.: Near-Earth interplanetary coronal mass ejections during solar cycle 23 (1996-2009): catalog and summary of properties. Sol. Phys. 264, 189 (2010). https://doi.org/ 10.1007/s11207-010-9568-6

Schmelz, J.T., Reames, D.V., von Steiger, R., Basu, S.: Composition of the solar corona, solar wind, and solar energetic particles. Astrophys. J. 755, 33 (2012). https://doi.org/10.1088/0004$637 \mathrm{X} / 755 / 1 / 33$

Sheeley Jr., N.R.: Surface evolution of the sun's magnetic field: a historical review of the fluxtransport mechanism. Living Rev. Sol. Phys. 2, 5 (2005). https://doi.org/10.12942/lrsp-2005-5

Wang, Y.-M., Pick, M., Mason, G.M.: Coronal holes, jets, and the origin of ${ }^{3}$ He-rich particle events. Astrophys. J. 639, 495 (2006). https://doi.org/10.1086/499355

Webb, D.F., Howard, T.A.: Coronal mass ejections: observations. Living Rev. Sol. Phys. 9, 3 (2012). https://doi.org/10.12942/lrsp-2012-3

Webber, W.R.: Solar and galactic cosmic ray abundances - a comparison and some comments. Proc. 14th Int. Cos. Ray Conf. Munich. 5, 1597 (1975) 
Open Access This chapter is licensed under the terms of the Creative Commons Attribution 4.0 International License (http://creativecommons.org/licenses/by/4.0/), which permits use, sharing, adaptation, distribution and reproduction in any medium or format, as long as you give appropriate credit to the original author(s) and the source, provide a link to the Creative Commons license and indicate if changes were made.

The images or other third party material in this chapter are included in the chapter's Creative Commons license, unless indicated otherwise in a credit line to the material. If material is not included in the chapter's Creative Commons license and your intended use is not permitted by statutory regulation or exceeds the permitted use, you will need to obtain permission directly from the copyright holder.

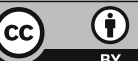

